\title{
Validation of a Wearable Sensor for Measuring Running Biomechanics
}

\author{
Rachel M. Koldenhoven Jay Hertel \\ Department of Kinesiology, University of Virginia, Charlottesville, VA, USA
}

\section{Keywords}

Bi-axial or tri-axial accelerometer devices - Gait · Monitor movement - Wearable physical activity monitoring $\cdot$ Wearables

\section{Abstract}

Background: Running biomechanics have traditionally been analyzed in laboratory settings, but this may not reflect natural running gait. Wearable sensors may offer an alternative. Methods: A concurrent validation study to determine agreement between the RunScribe ${ }^{\mathrm{TM}}$ wearable sensor (triaxial accelerometer and gyroscope) and the 3D motion capture system was conducted. Twelve injury-free participants ( 6 males, 6 females; age $=23.1 \pm 5.5$ years, weekly mileage $=16.1 \pm 9.3)$ ran 1.5 miles on a treadmill. Ten consecutive strides from each limb were collected, and the mean values were analyzed. Pronation excursion, maximum pronation velocity, contact time, and cycle time were compared between measurement platforms using intraclass correlation coefficients (ICC) and Bland-Altman analyses. Results: Excellent ICC estimates were found for maximum pronation velocity, contact time, and cycle time. Pronation excursion demonstrated fair ICC estimates. The mean differences between platforms were small with limits of agreement clustered around zero, except for contact time measures which were consistently higher with the RunScribe compared to the camera-based system. Conclusion: Our study revealed that the RunScribe wearable device showed good to excellent concurrent validity for maximum pronation velocity, contact time, and cycle time; however, direct comparisons or results between the two platforms should not be used. 
Koldenhoven and Hertel: Validation of a Wearable Sensor for Measuring Running Biomechanics

\section{Introduction}

Traditionally, running gait for healthy and injured individuals has been measured in a laboratory setting; however, the results of these studies may lack generalizability to natural running. Many runners train in an outdoor environment and do not regularly run in a laboratory or under direct observation. Laboratory studies typically analyze only 5-15 steps of a subject's given run due to constraints in data storage and analysis, but individuals may take roughly 2,000 steps per mile. Due to potential changes in running mechanics over long runs, analyzing an abundance of steps may be beneficial. The utility of wearable sensors to measure gait biomechanics may be useful for both clinicians working with runners to monitor them over several runs and for researchers studying a variety of runners.

Wearable sensors may allow for continuous measurement of gait mechanics across thousands of steps during a distance runner's typical training run and, thus, may better monitor various aspects of gait biomechanics by capturing information about each step [1, 2]. Accelerometers are the most widely used and accepted type of sensor for measuring physical activity outside of traditional laboratory settings; however, running biomechanics are vastly understudied using wearable sensors [3]. Good to excellent test-retest reliability (interclass correlation coefficient (ICC) $=0.70-0.97$ ) has been found for accelerometers used in gait assessment when compared against the gold standard 3D motion analysis system [4]. In a study looking at concurrent validity of an accelerometer to the GAITRite ${ }^{\circledR}$ electronic walkway in Parkinson's disease patients, good to excellent reliability (ICC $=0.76-0.99$ ) was found for gait speed, step length, and step frequency [5]. Such data are lacking in higher functioning distance runners.

The RunScribe ${ }^{\mathrm{TM}}$ (Scribe Labs, Inc., Half Moon Bay, CA, USA) is a small wearable device that has been marketed for measuring running gait biomechanics and can be worn on the runner's shoe. This wearable device captures kinematic, kinetic, and spatiotemporal data of each individual step and poses great advantage over traditional pedometers and accelerometers. Additionally, the runner cannot feel the sensor as it is placed directly on the shoe. This placement allows them to run freely compared to the traditional laboratory set-up when markers or sensors are attached to the individual. Our purpose was to determine the concurrent validity of the RunScribe to the gold standard 3D motion capture system.

\section{Methods}

We performed a concurrent validation study comparing RunScribe wearable sensors to a 3D motion capture system during simultaneous collection of kinematic (maximum pronation velocity and pronation excursion) and spatiotemporal (contact time and cycle time) variables during treadmill running.

\section{Subjects}

Twelve recreational runners ( 6 males, 6 females; age $=23.1 \pm 5.5$ years, weekly mileage $=16.1 \pm 9.3$ ) free of lower-extremity musculoskeletal injuries within the past 12 months participated. Informed consent was obtained from all participants, and the study protocol was approved by the university's Institutional Review Board.

\section{Instrumentation}

A 12-camera motion capture system (Vicon Motion Systems, Inc., Lake Forest, CA, USA) with a sampling rate of $250 \mathrm{~Hz}$ integrated with an instrumented treadmill (Bertec, Columbus, $\mathrm{OH}, \mathrm{USA}$ ) sampling at $1,000 \mathrm{~Hz}$ were synchronized. Kinematic data were exported using 
Koldenhoven and Hertel: Validation of a Wearable Sensor for Measuring Running Biomechanics

Motion Monitor software (Innovative Sports Training, Chicago, IL, USA). All participants wore standardized laboratory shoes (Brooks Defyance; Brooks Sports, Inc., Bothell, WA, USA).

The RunScribe wearable sensors contained a triaxial accelerometer and gyroscope with onboard processing and memory capabilities and sampled at $200 \mathrm{~Hz}$.

\section{Procedures}

Ten clusters consisting of 42 retroreflective markers were placed on the participants' posterior thorax, sacrum, and bilaterally on the lateral midthigh, lateral midshank, dorsum of the forefoot, and posteriorly on the calcaneus (extending through a cutout in the shoe). Segments were digitized for the left and right anterior superior iliac spine, C7/T1, T12/L1, L5/S1, medial and lateral knee joint lines bilaterally, and bilaterally for the medial and lateral malleoli to determine joint centers. All participants completed a 5-minute familiarization period of running on the treadmill and were instructed to verbalize when running speed was comfortable and attainable for 1.5 miles of continuous running.

Following the familiarization period, the wearable sensors were placed on the back of each shoe. The participants ran on the treadmill for 1.5 miles at their preferred speed, while data were collected simultaneously by the measurement systems. Motion capture data were collected for $60 \mathrm{~s}$ when the participant reached the 1-mile mark.

\section{Data Processing}

Kinematic data from the motion capture system were reduced to 101 data points per step representing $0-100 \%$ of the gait cycle. Initial contact was identified when the ground reaction force exceeded $20 \mathrm{~N}$. Toe-off was identified when the vertical ground reaction force became $0 \mathrm{~N}$. The time from initial contact to toe-off was defined as the contact time, and the time from initial contact to following ipsilateral initial contact was defined as cycle time. Maximum rearfoot eversion velocity was defined as the maximum velocity during the stance phase for rearfoot eversion.

RunScribe data were transferred from the device to the mobile app via Bluetooth and uploaded to the company website via wireless internet. Then, the data were downloaded from the website for analysis. The steps were matched from both systems based on the timestamp. Ten consecutive strides for each limb from both platforms were analyzed for each participant. All data were exported to Microsoft Excel version 15.33 (Microsoft Corp., Redmond, WA, USA) for further data processing.

\section{Statistical Analysis}

ICC $_{3,1}$ and corresponding standard error of measure were used to analyze the reliability and precision of measures between the RunScribe and motion capture systems using SPSS version 22.0 (SPSS, Inc., Chicago, IL, USA). Bland-Altman plots were also created to assess the mean difference and associated 95\% limits of agreement between the two measurement systems.

\section{Results}

The results are detailed in Table 1 . For maximum pronation velocity, contact time, and cycle time, we found good (ICC $=0.70-0.89$ ) to excellent (ICC $>0.90$ ) reliability estimates, and for pronation excursion, we found fair (ICC $=0.40-0.57$ ) reliability estimates. The mean differences were typically small with limits of agreement clustered around zero, with the exception of contact time measures which were consistently higher with the RunScribe compared to the camera system (online suppl. material; for all online suppl. material, see www.karger.com/doi/10.1159/000491645). 
Koldenhoven and Hertel: Validation of a Wearable Sensor for Measuring Running Biomechanics

Table 1. Values from the RunScribe ${ }^{\mathrm{TM}}$ and 3D motion capture system for right and left limbs and results for mean differences, intraclass correlation coefficients, and standard error of measurement values between the two platforms

\begin{tabular}{|c|c|c|c|c|c|c|}
\hline & $\begin{array}{l}\text { Maximum } \\
\text { pronation } \\
\text { velocity, } \% \text { s }\end{array}$ & $\begin{array}{l}\text { Pronation } \\
\text { excursion, }^{\circ}\end{array}$ & $\begin{array}{l}\text { Contact time, } \\
\text { ms }\end{array}$ & $\begin{array}{l}\text { Cycle time, } \\
\text { ms }\end{array}$ & $\begin{array}{l}\text { Stride length, } \\
\mathrm{m}\end{array}$ & $\begin{array}{l}\text { Stride pace, } \\
\mathrm{m} / \mathrm{s}\end{array}$ \\
\hline \multicolumn{7}{|l|}{ Left limb } \\
\hline RunScribe $^{\mathrm{TM}}$ & $444 \pm 177$ & $10.6 \pm 5.3$ & $292 \pm 25$ & $727 \pm 27$ & $2.3 \pm 0.3$ & $2.9 \pm 0.4$ \\
\hline 3D motion capture & $436 \pm 169$ & $14.6 \pm 7.6$ & $264 \pm 22$ & $719 \pm 28$ & $3.0 \pm 0.3$ & $2.7 \pm 0.1$ \\
\hline Mean difference & 8.6 & -4.0 & 27.8 & 8.1 & 0.7 & 0.1 \\
\hline LOA & $-165.4,182.6$ & $-16.1,8.1$ & $9.8,45.9$ & $-11.6,27.7$ & $-1.4,0.1$ & $-0.2,0.3$ \\
\hline ICC & 0.74 & 0.57 & 0.93 & 0.94 & 0.86 & 0.43 \\
\hline SEM & 88.2 & 4.2 & 6.2 & 6.7 & 0.1 & 0.2 \\
\hline \multicolumn{7}{|l|}{ Right limb } \\
\hline RunScribe $^{T M}$ & $510 \pm 217$ & $13.1 \pm 5.8$ & $298 \pm 23$ & $726 \pm 26$ & $2.2 \pm 0.2$ & $3.1 \pm 0.3$ \\
\hline 3D motion capture & $361 \pm 188$ & $13.7 \pm 8.9$ & $269 \pm 22$ & $723 \pm 28$ & $3.0 \pm 0.1$ & $2.7 \pm 0.1$ \\
\hline Mean difference & 149.3 & 0.5 & 29.1 & 3.2 & 0.8 & 0.4 \\
\hline LOA & $-145.8,444.4$ & $-17.1,16.0$ & $11.5,46.6$ & $-20.3,26.8$ & $-1.2,-0.5$ & $-0.1,0.8$ \\
\hline ICC & 0.87 & 0.4 & 0.92 & 0.91 & 0.8 & 0.73 \\
\hline SEM & 73.0 & 5.7 & 6.4 & 8.1 & 0.1 & 0.1 \\
\hline
\end{tabular}

Values are given as means \pm SD unless indicated otherwise.

ICC, intraclass correlation coefficient; LOA, limits of agreement; SD, standard deviation; SEM, standard error of measurement.

\section{Discussion}

Our study revealed that the RunScribe wearable device showed good to excellent concurrent validity for maximum pronation velocity, contact time, and cycle time. Kinematic and spatiotemporal measures were highly correlated between the wearable sensor and the motion capture system; however, the absolute values of measures across the two platforms were not identical. The RunScribe provides additional insight to running mechanics compared to traditional accelerometers or pedometers, but the results should be interpreted with caution and should not be compared with laboratory-based studies.

In the frontal plane, the RunScribe underestimated pronation excursion by up to $4^{\circ}$ and overestimated the maximum pronation velocity by up to $150^{\circ}$ s. Additionally, the limits of agreement were quite large for both measures suggesting further differences between the measurement devices. This discrepancy between measures may also be problematic because previous studies have suggested that excessive ranges (too low or too high) in pronation excursion and pronation velocity may lead to increased injury risk [6-8]. The RunScribe was not able to assess pronation measures as accurately as the gold standard motion capture. Therefore, it does not appear that direct comparisons of biomechanical measures should be made across measurement platforms for pronation measures.

Additionally, the RunScribe was able to accurately measure cycle time but typically overestimated the contact time by about $10 \%$. Measuring these outcomes through a wearable device could allow for the observation of runners over time and in a natural environment, but they, then, need to be interpreted with caution. Spatiotemporal variables have been easily manipulated in gait-training studies by instructing patients to "run softer" or to increase their step rate $[9,10]$. These strategies have been used in injured populations and successfully reduced impact forces and altered gait patterns [10]. The RunScribe may be a reasonable tool to use for measuring spatiotemporal variables throughout runs and to track changes across several runs. 
Koldenhoven and Hertel: Validation of a Wearable Sensor for Measuring Running Biomechanics

Limitations in our study include that the RunScribe device collects data onboard, and, then, the data are run through a proprietary algorithm, so the exact formulae are unknown. We compared the RunScribe data to variables that we defined within the motion capture system. The data from both systems may not be directly comparable as we do not know exactly how the RunScribe variables were calculated. Discrepancies may also be present due to the nature of treadmill running. This study was performed in a laboratory on a treadmill as an initial form of device validation. The runners maintained a consistent speed throughout the run so the distance could be calculated and steps between platforms could be matched. Future research should consider validating the device in a field setting at different speeds and with surface types outside of a controlled laboratory setting.

\section{Conclusion}

Our study revealed that the RunScribe wearable device showed good to excellent concurrent validity for maximum pronation velocity, contact time, and cycle time. Although the systems were not in perfect agreement, clinicians and researchers may find this wearable sensor useful in their assessment of distance running for kinematic and spatiotemporal measures.

\section{Statement of Ethics}

Informed consent was obtained from all participants, and the study protocol was approved by the university's Institutional Review Board.

\section{Disclosure Statement}

There are no potential conflicts of interest in regard to this manuscript.

\section{References}

1 Diaz KM, Krupka DJ, Chang MJ, et al: Fitbit ${ }^{\circledR}$ : an accurate and reliable device for wireless physical activity tracking. Int J Cardiol 2015;185:138-140.

2 Kim A, Kim J, Rietdyk S, Ziaie B: A wearable smartphone-enabled camera-based system for gait assessment. Gait Posture 2015;42:138-144.

3 Chen KY, Janz KF, Zhu W, Brychta RJ: Re-defining the roles of sensors in objective physical activity monitoring. Med Sci Sports Exerc 2012;44(1 suppl 1):S13-S23.

4 Chung PYM, Ng GYF: Comparison between an accelerometer and a three-dimensional motion analysis system for the detection of movement. Physiotherapy 2012;98:256-259.

5 Lord S, Rochester L, Baker K, Nieuwboer A: Concurrent validity of accelerometry to measure gait in Parkinsons Disease. Gait Posture 2008;27:357-359.

6 Ferber R, Hreljac A, Kendall KD: Suspected mechanisms in the cause of overuse running injuries. Sports Health 2009;1:242-246.

7 Messier S, Davis S, Curl W, Lowery R, Pack R: Etiologic factors associated with patellofemoral pain in runners. Med Sci Sports Exerc1991;23:1008-1015.

8 Rolf C: Overuse injuries of the lower extremity in runners. Scand J Med Sci Sports 1995;5:181-190.

9 Davis IS, Futrell E: Gait retraining: altering the fingerprint of gait. Phys Med Rehabil Clin N Am 2016;27:339355.

10 Crowell HP, Davis IS: Gait retraining to reduce lower extremity loading in runners. Clin Biomech 2011;26: 78-83. 